Case Reports

\title{
Consumption of an Extremely Biodiverse Probiotic and a Supplement based on Microbial Chondroitin Sulfate is Associated with Very Low Serum Alpha-N- acetylgalactosaminidase (Nagalase) Activity and Decrease of C-reactive Protein Values
}

\author{
${ }^{1,2,3}$ Michael Carter, ${ }^{4}$ Stefania Pacini and ${ }^{4}$ Marco Ruggiero \\ ${ }^{1}$ Real Health Clinics, Atlanta, GA, USA \\ ${ }^{2}$ Health Revival Partners, USA \\ ${ }^{3}$ Tissue Regeneration Technologies, Woodstock, GA, USA \\ ${ }^{4}$ Silver Spring Sagl, Mendrisio, Switzerland
}

Article history

Received: 06-02-2020

Revised: $16-02-2020$

Accepted: 20-04-2020

Corresponding Author: Marco Ruggiero

Silver Spring Sagl, Via

Raimondo Rossi 24, Mendrisio 6850, Switzerland

Email: info@bravo-europe.com

\begin{abstract}
We describe the case of a 57 -years-old male healthy professional who added to his nutritional plan a novel, extremely biodiverse, probiotic and a supplement based on microbial chondroitin sulfate, vitamin $\mathrm{D}_{3}$ and ultrapure phosphatidylcholine to improve his healthy life expectancy. After three months of such a nutritional regimen, serum alpha-Nacetylgalactosaminidase (nagalase) activity was 0.40 that is a value below the minimum level established for adults (normal values: 0.50-0.95 $\mathrm{nMol} / \mathrm{mL} / \mathrm{min}$ ). C-reactive Protein (CRP) was less than 1 (normal values: $0.00-5.00 \mathrm{mg} / \mathrm{L}$ ), a value significantly lower than that recorded before this experience. At the end of the observation period, serum albumin level was $45 \mathrm{~g} / \mathrm{L}$, a value very close to the maximum reference value (normal values: 32.00-46.00 g/L). The Prognostic Inflammatory Nutritional Index (PINI) score was 0.05 , a value that is close to the minimum calculated value (normal values: $0.00-1.00$ ) and suggests a very low risk of death for all causes. Observation in silico and experiments in vitro demonstrated that both the probiotic and the supplement formed complexes with human nagalase with an efficiency 100 fold higher than that of purified Gc proteinderived Macrophage Activating Factor (GcMAF). The collection of these observations suggests that the combination of this probiotic and this supplement may lead to overall beneficial effects on health as evidenced by positive changes of indicators of immune system function and healthy aging such as nagalase, CRP, albumin and PINI score.
\end{abstract}

Keywords: Nagalase, GcMAF, Probiotic, Colostrum, Phages, Chondroitin Sulfate, Vitamin $\mathrm{D}_{3}$, Phosphatidylcholine, C-reactive Protein, Aging

\section{Introduction}

Serum alpha-N-acetylgalactosaminidase (nagalase) and C-reactive Protein (CRP) are two markers of chronic conditions that have been used to monitor a variety of diseases as well as to predict successful aging and healthy life expectancy. Nagalase, an enzyme, was first proposed as a marker for cancer and viral infections and its increased serum activity in these conditions was associated with immune system deficiency since nagalase prevents the formation of Gc protein-derived Macrophage Activating Factor (GcMAF), an immune stimulant cytokine (Yamamoto and Naraparaju, 1997; Yamamoto and Urade, 2005; Yamamoto, 2006). This occurs because nagalase breaks the bond between alpha-Nacetylgalactosamine and the amino acid threonine situated in the third domain of Gc protein (the precursor of GcMAF), thus effectively removing alpha-Nacetylgalactosamine that is considered the active site of GcMAF. Elevated serum nagalase activity has been reported in a variety of tumors ranging from experimental squamous cell carcinomas to human malignant melanomas (Korbelik et al., 1998; Greco et al., 2009); consistent with these observation, successful 
immunotherapy of cancer was associated with a decrease of serum nagalase activity (Thyer et al., 2013a; Schwalb et al., 2016). The significance of nagalase in oncology is well exemplified by the title of a recent review that reads "Is $\alpha$ $\mathrm{N}$-acetylgalactosaminidase the key to curing cancer?" (Saburi et al., 2017). However, elevated serum nagalase activity is associated also with non-neoplastic conditions ranging from bacterial infection (Caines et al., 2008) to autism (Bradstreet et al., 2012). Interestingly, in autism, just like in cancer, successful immunotherapy was associated with significant decrease of serum nagalase activity, thus lending credibility to the hypothesis that elevated nagalase activity has to be considered more a pathogenetic factor for those conditions rather than a simple marker.

If the literature on nagalase is relatively new and limited to a small, albeit significant, number of peerreviewed papers, the literature on CRP, an acute-phase protein that increases in the presence of infectious and inflammatory processes, is older and ampler and refers to a large number of conditions ranging from cancer (Allin and Nordestgaard, 2011) to autism (Brown et al., 2014), depression (McFarland et al., 2019), cardiovascular diseases (Cozlea et al., 2013), multiple sclerosis (Dowling and Cook, 1976) and anemia (Breiterman-White, 2006) to name a few. In a study on incurable cancer patients published in 2012, we demonstrated that CRP, integrated into the context of the Prognostic Inflammatory Nutritional Index (PINI) score, was a powerful marker for predicting overall survival (Fabris et al., 2012). Similar to nagalase, CRP may bear more pathogenic significance than simply being a marker; according to a recent paper "CRP is pathogenic in a number of diseases including hypertensive cardiovascular and kidney complications, diabetic nephropathy and acute and chronic kidney diseases" (Tang et al., 2017). Because of this, low levels of CRP have been proposed also as determinants for successful aging (Puzianowska-Kuźnicka et al., 2016). As far as aging is specifically concerned, Ferrucci et al. (2005) demonstrated that CRP increases 3-5 fold during aging in women and men, respectively. Oluleye et al. (2013) demonstrated that risk of death for all causes was significantly reduced when CRP was below $3 \mathrm{mg} / \mathrm{L}$ when compared with values above $3 \mathrm{mg} / \mathrm{L}$ in more than 11,000 subjects whose average age was 63 . Therefore, it can be deduced that strategies aimed at lowering serum CRP levels are to be associated with reduced risk of allcause mortality, increased longevity and successful aging, as proposed by Arima et al. (2008).

To increase his potential for healthy longevity, one of the authors of this article, Dr. MC, a Medical Doctor, decided to implement a nutritional strategy aimed at lowering serum nagalase activity and CRP levels and improving other markers of successful aging. This strategy comprised a novel supplement containing lowmolecular-weight microbial chondroitin sulfate, ultrapure phosphatidylcholine and vitamin $\mathrm{D}_{3}$, (imuno ${ }^{\circledR}$, imuno Corporation, Vanuatu) and an extremely diverse probiotic formula (Freeze-Dried Bravo Colostrum and Probiotic Complex; Silver Spring Sagl, Mendrisio, Switzerland, here abbreviated in PMF - Product of Microbial Fermentation). Composition of PMF in terms of probiotic microbes, phages and plasmids has been recently described in full detail (Pacini and Ruggiero, 2019a; 2019b). The supplement used by Dr. MC $\left(\right.$ imuno $^{\circledR}$ ) represents the latest evolution of the concept of GcMAF and its mechanism of action and indications have been thoroughly described in peer-reviewed publications (Ruggiero and Pacini, 2018a; 2018b; Antonucci et al., 2018; 2019a; 2019b).

Here, we describe the results observed by Dr. MC after three months of assumption of imuno ${ }^{\circledR}$ and the PMF and we present a collection of in silico and in vitro observations highlighting the molecular mechanisms of action responsible for these results.

\section{Materials and Methods}

Activity of nagalase in serum was determined at R.E.D. Laboratories (Zellik, Belgium) through a proprietary method consisting of a two-step immunecapture assay. Nagalase activity in serum is measured kinetically through conversion of a fluorogenic substrate in function of time. The test is standardized against a large serum pool of carefully selected healthy subjects with normal white blood cell count, no laboratory signs of inflammation, CRP less than $1 \mathrm{mg} / \mathrm{L}$ and no clinical history of immune disease of diabetes. Nagalase activity measurement in healthy subjects has allowed to establish a normal range of substrate conversion between 0.50 and $0.95 \mathrm{nMol} / \mathrm{mL} / \mathrm{min}$ for adults.

Study of in vitro interaction between GcMAF, imuno $^{\circledR}$, or PMF and human nagalase was also performed by R.E.D. Laboratories (Zellik, Belgium) where GcMAF was purified. The experiments were performed using microtiter plates coated with a specific antibody able to capture human nagalase. Samples were incubated with a standardized dilution of a pool of 300 human sera from healthy subjects identified as described above and, after $1 \mathrm{~h}$ incubation and exhaustive washing, complexes formed by nagalase and purified GcMAF, used as positive control, or nagalase and imuno ${ }^{\circledR}$, or nagalase and PMF, were detected with a horse radish peroxidase conjugate of a rabbit antibody. In order to establish the kinetics of interaction between nagalase and GcMAF, nagalase and imuno ${ }^{\circledR}$, or nagalase and PMF, the serum pool was mixed either with $200 \mathrm{ng}$ of purified GcMAF, or with two dilutions $(1: 50$ and $1: 100)$ of imuno $^{\circledR}$, or with two dilutions $(1: 10$ and $1: 100)$ of PMF in phosphate buffered saline (PBS). The mixture was then incubated at room temperature for 4, 24, 48, 72 and $120 \mathrm{~h}$. 
Values for nagalase complex forming activity in the absence of GcMAF, imuno ${ }^{\circledR}$ or PMF, that is with only PBS in the reaction mixture, were taken as 1.00 . The experiment was repeated twice and the results reported in Figs. 3-7 are the means of the two experiments.

As far as other markers of health are concerned, determination of nagalase activity in serum was complemented by evaluation of another serum enzyme that is useful as cancer and inflammation marker, dipeptidyl peptidase-4 (DPP4) also known as CD26 (Pro and Dang, 2004; Ghorpade et al., 2018). This is an enzyme expressed on the surface of most cell types, including macrophages and T-lymphocytes and is associated with immune regulation, signal transduction and apoptosis (Havre et al., 2008; Hiromura et al., 2018). The PINI score was calculated as described by Fabris et al. (2012).

Since this is a single case report that does not produce generalizable knowledge, nor is it an investigation of an FDA regulated product, an Institutional Review Board (IRB) review is not required for this activity. Likewise, since this case report describes the experience of one of the Authors, Dr. MC, the concept of "informed consent" is implicit in the authorship of the study.

\section{Case Presentation}

Dr. MC, Medical Doctor, is an active and energetic professional born in 1962, who works as Corporate Medical Director of Real Health Clinics in Atlanta, GA, as Chief Medical Officer for Health Revival Partners and as Chief Medical Advisor for Tissue Regeneration Technologies, Woodstock, GA, USA; he added imuno ${ }^{\circledR}$ $(0.1 \mathrm{~mL}$ twice a week) and PMF (1 capsule per day) to his nutritional regimen without any other change to his nutrition or lifestyle. His experience lasted for three months and ended in July 2019. Blood analyses were performed immediately after the three-month experience.

After three months of consumption of imuno ${ }^{\circledR}$ and $\mathrm{PMF}$, nagalase activity, expressed as $\mathrm{nMol} / \mathrm{mL} / \mathrm{min}$, was 0.40 that is a value below the minimum level established for adults (normal values: 0.50-0.95). Consistent with this result, DPP4 activity was $24 \mathrm{nMol} / \mathrm{mL} / \mathrm{min}$ (normal values: 18.80-33.79), further indicating optimal functioning of the immune system. CRP, expressed as $\mathrm{mg} / \mathrm{L}$, was less than 1 (normal values: $0.00-5.00$ ). It is worth considering that a previous blood analysis, performed in November 2018 at Lab Corp (Virtual Clinics, Terre Haute, IN, USA) evidenced a CRP value of $0.29 \mathrm{mg} / \mathrm{L}$ that, although within the normal value reference, was significantly higher. At the end of the observation period, serum albumin level was $45 \mathrm{~g} / \mathrm{L}$, a value very close to the maximum reference value (normal values: $32.00-46.00 \mathrm{~g} / \mathrm{L}$ ). It is worth noticing that serum albumin levels physiologically decrease with aging and lower levels are associated with increased risk of all-cause mortality (Weaving et al., 2016). Consistent with the high albumin/low CRP values, the PINI score was 0.05 a value that is close to the minimum calculated value (normal values: $0.00-1.00$ ) and suggests a very low risk of death for all causes.

\section{In silico Observation and Experimental Results in vitro}

Having observed a significant decrease of serum nagalase activity following assumption of imuno ${ }^{\circledR}$ and PMF, we performed studies in silico and in vitro to establish the molecular mechanisms of action responsible for the observation of Dr. MC. First, we sought to determine the potential sites of interaction between nagalase and GcMAF, nagalase and imuno ${ }^{\circledR}$, or nagalase and PMF using the "Align" tool of Uniprot, a tool designed to "align two or more protein sequences with the Clustal Omega program to view their characteristics alongside each other" (www.uniprot.org). We chose the sequence P02774 VTDB_HUMAN for human GcMAF and the sequence P17050 NAGAB_HUMAN for human nagalase. Figure 1 shows the binding sites of human nagalase as indicated by green arrows. The active sites are indicated by red arrows; the aspartic acid in the sequence DDIQ is indicated by a green and a red arrow since it is both a binding site and the second active site of the enzyme. The binding and active sites were identified by the Align tool of Uniprot. Figure 1 also shows the active site of GcMAF that is encased in a square with black outline and indicated by a black arrow. The sequence TPTELAK is the sequence where alpha-N-acetylgalactosamine is attached to the amino acid threonine. In the inactive form of GcMAF, that is in its precursor, sialic acid and D-galactose are attached to alpha- $\mathrm{N}$-acetylgalactosamine -that is the active site of GcMAF - thus masking it and preventing interaction with its receptor. When sialic acid and D-galactose are removed, alpha-N-acetylgalactosamine can interact with its receptor and GcMAF is active. Nagalase breaks the bond between alpha-N-acetylgalactosamine and the amino acid threonine in the sequence TPTELAK only when GcMAF is in its inactive form, that is when sialic acid and $\mathrm{D}$-galactose are attached to alpha-N-acetylgalactosamine. It is worth noticing that the binding and active sites of nagalase are flanked by stretches of hydrophobic amino acids that are highlighted in indigo in Fig. 1. The active site of GcMAF comprises hydrophobic amino acids and it is also flanked by hydrophobic amino acids. Based on the molecular model we first proposed in 2013 (Thyer et al., $2013 \mathrm{~b})$ these hydrophobic interactions are deemed responsible for formation of complexes between nagalase and GcMAF. In PMF, such an interaction is strengthened by the presence of fatty acids that are natural components of milk and colostrum; enhancement of hydrophobic interactions may be one of the factors responsible for the 100 fold higher nagalase complex forming activity observed in PMF as compared to purified GcMAF (Figs. 57). It may be worth noticing that the stretches of hydrophobic amino acids flanking the binding and active 
sites of nagalase may also bind the ultrapure phosphatidylcholine that forms ordered structures of phospholipid bilayers in cell-like membranes comprising the tri-dimensional molecular structure of imuno ${ }^{\circledR}$ (Ruggiero and Pacini 2018a; 2018b). In analogy with PMF, these enhanced hydrophobic interactions may be responsible for the 100 fold higher nagalase complex forming activity observed in imuno ${ }^{\circledR}$ as compared to purified GcMAF (Figs. 3, 4 and 7).

Figure 2 shows the distribution of positively (highlighted in green) and negatively (highlighted in red) charged residues in GcMAF and nagalase with particular reference to the binding and active sites of both proteins that are indicated by squares outlined in black and black arrows. Both active sites of nagalase are constituted by negatively charged residues (aspartic acid). Figure 2 also shows how the active sites of nagalase present alternation of positively and negatively charged residues that may represent the binding sites for the highly sulfated low-molecular-weight microbial chondroitin sulfate of imuno ${ }^{\circledR}$ that is constituted by moieties alternating negative and positive charges. More specifically, electrostatic interaction between the aspartic acid residues of the active sites of nagalase and the alpha- $\mathrm{N}$-acetylgalactosamine that is a constituent of chondroitin sulfate, complementing the hydrophobic interactions described above, may explain the 100 fold higher nagalase complex forming activity observed in imuno $^{\circledR}$ as compared to purified GcMAF (Figs. 3, 4 and 7). In other words, here we demonstrate that the alpha-Nacetylgalactosamine of chondroitin sulfate works at the molecular level like the active site of GcMAF with the notable difference that there is only one alpha-Nacetylgalactosamine moiety per GcMAF molecule whereas there are 50 to 100 alpha-N-acetylgalactosamine moieties per chondroitin sulfate molecule. In addition, the peculiar conformation of imuno ${ }^{\circledR}$ with negatively charged surfaces constituted by carboxyl and sulfonic groups encased in a phospholipid bilayer, may be involved in the binding and electrostatic neutralization of protein inserts characterized by high density of positively charges residues. In particular, it may be involved in binding the sequences TNGTKR, HKNNKS, RSYLTPGDSSSG and QTNSPRRA that are characterized by a high surface concentration of positive charges interspersed with hydrophobic residues (Zunaid et al., 2020). It is worth noticing that neutralization of positively charged residues may represent a strategy that exploits electrostatic interactions between charges on the surface of bioactive molecules.
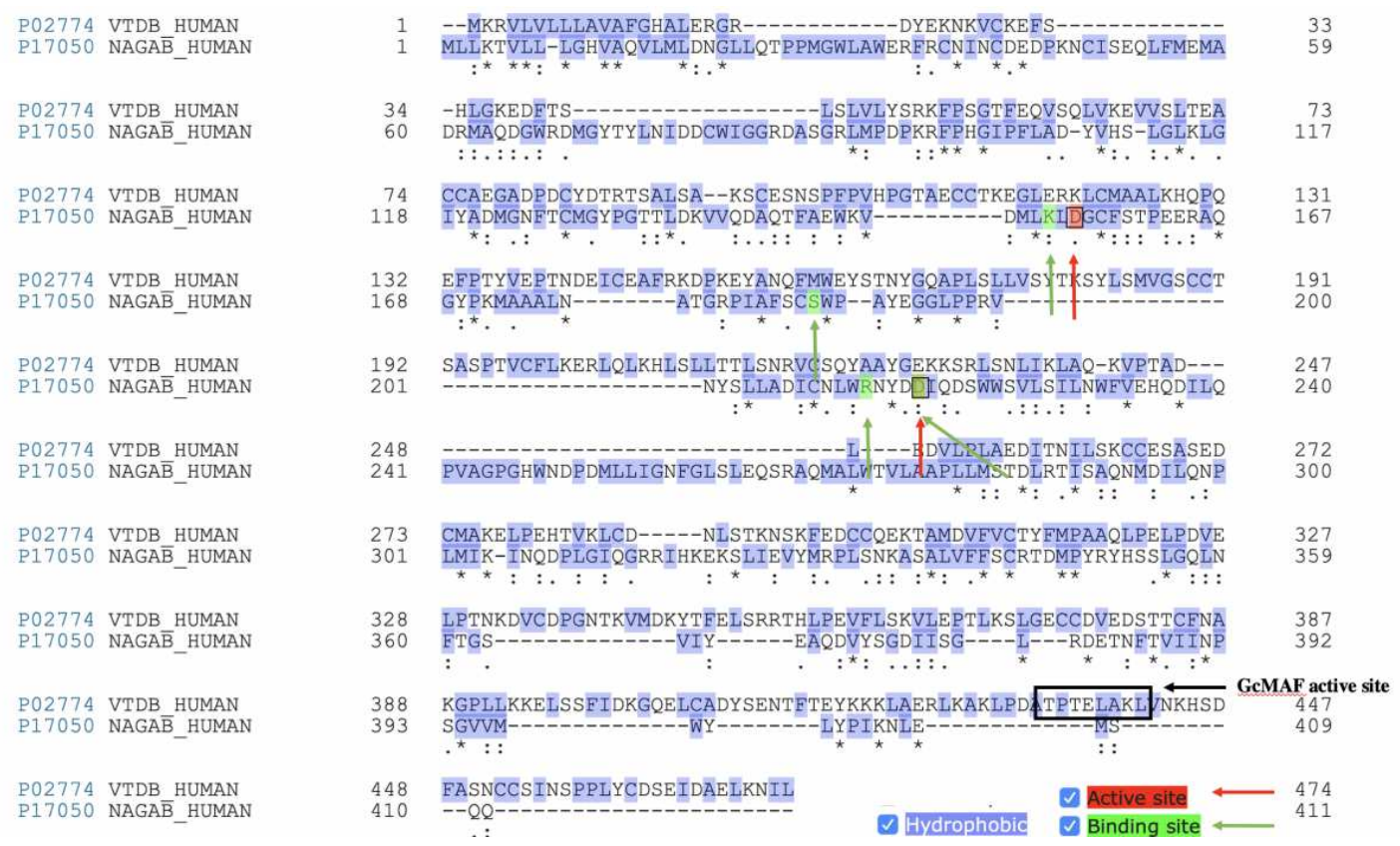

Fig. 1: Sequence alignment between Gc protein-derived macrophage activating factor, GcMAF and nagalase. The sequences of GcMAF (P02774 VTDB_HUMAN) and nagalase (P17050 NAGAB_HUMAN) were obtained and aligned using the Align tool of Uniprot (uniprot.org). The conventional consensus symbols are: "*" indicating that the residues are identical in all sequences in the alignment. ":" indicating that conserved substitutions have been observed. "." indicating that semi-conserved substitutions are observed, that is, amino acids having similar features. Hydrophobic amino acids are highlighted in indigo. The binding sites of nagalase are indicated by green arrows. The active sites are indicated by red arrows; the aspartic acid in the sequence DDIQ is indicated by a green and a red arrow since it is both a binding site and the second active site of the enzyme. The active site of GcMAF is encased in a square with black outline and indicated by a black arrow. The sequence TPTELAK is the sequence where alpha-N-acetylgalactosamine - that is the substrate of nagalase - is attached to the amino acid threonine 


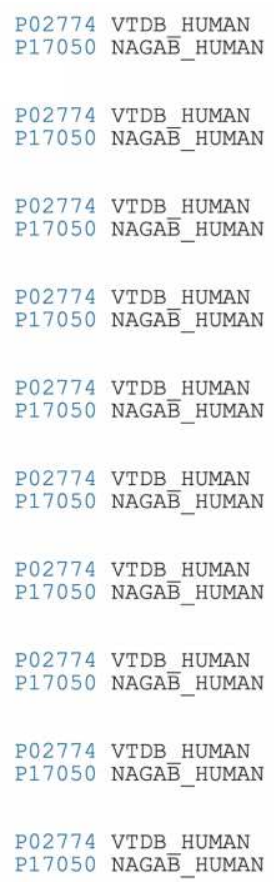

192

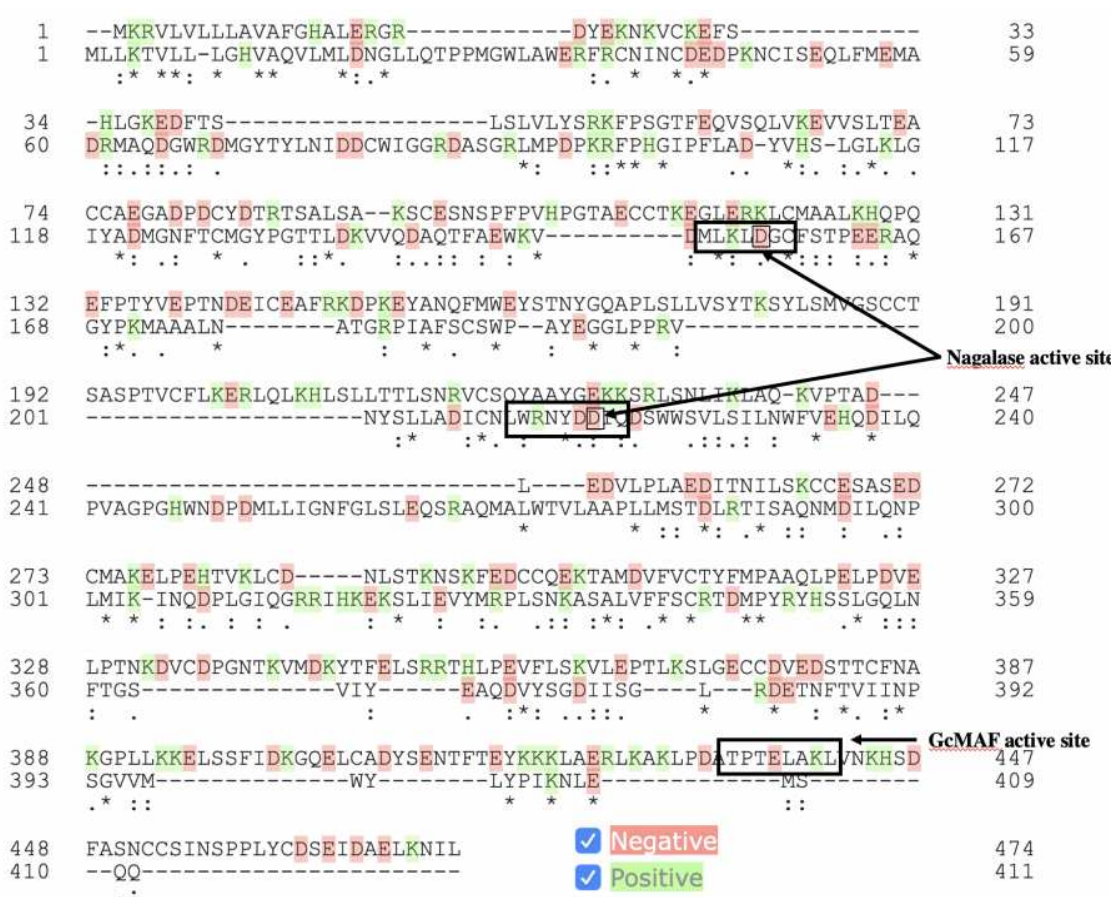

Fig. 2: Sequence alignment between Gc protein-derived macrophage activating factor, GcMAF and nagalase. Negatively and positively charged amino acids. Positively (highlighted in green) and negatively (highlighted in red) charged residues in GcMAF and nagalase are depicted, with particular reference to the binding and active sites of both proteins that are encased in squares outlined in black and indicated by black arrows

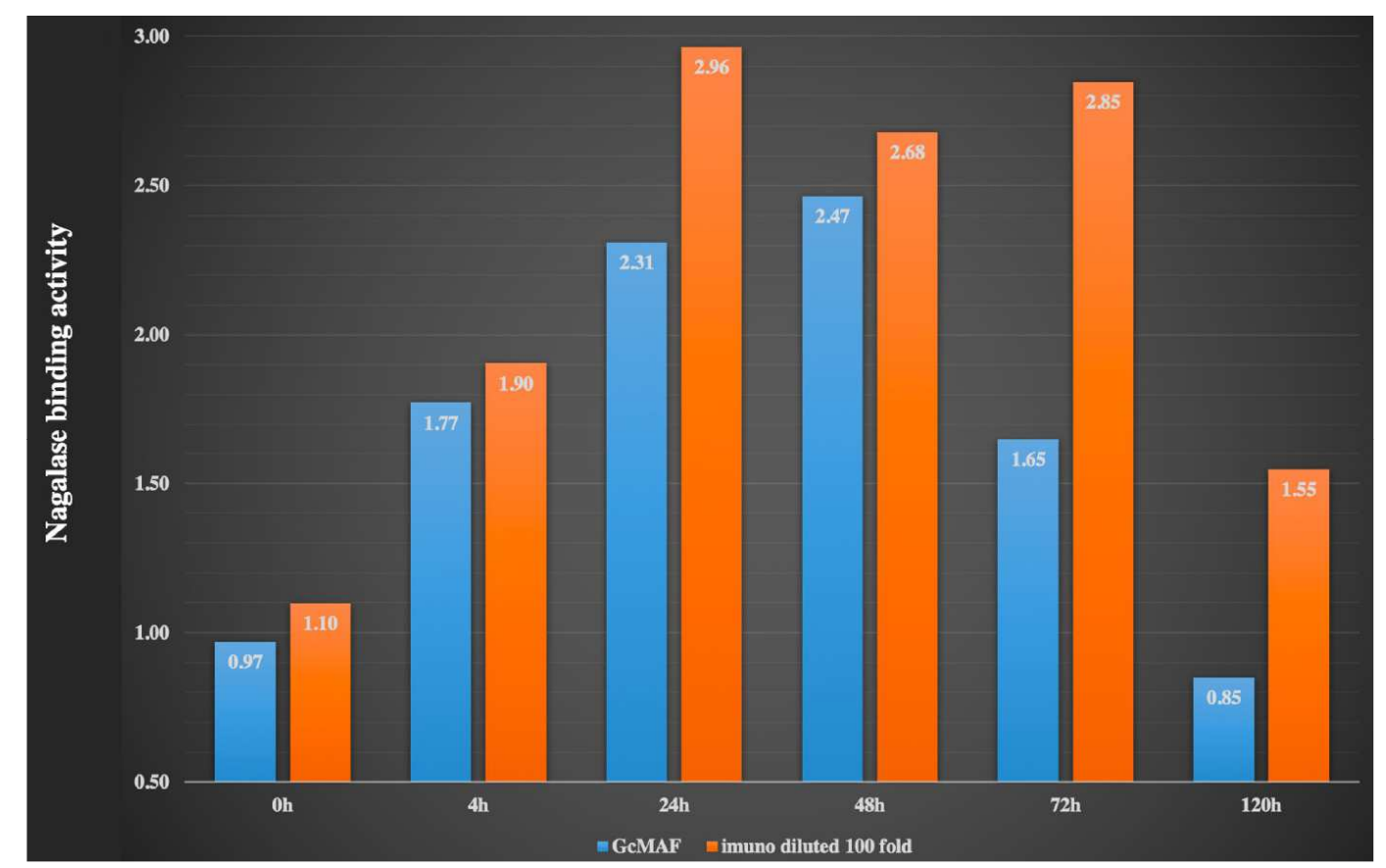

Fig. 3: Kinetics of complex formation between purified GcMAF or imuno ${ }^{\circledR}$ and human nagalase. Values for nagalase complex forming activity in the absence of GcMAF or imuno ${ }^{\circledR}$, with only PBS in the reaction mixture, were taken as 1.00 . imuno ${ }^{\circledR}$, diluted 100 fold, had an initial value (at time 0) higher than that observed with PBS alone, thus demonstrating immediate, intrinsic GcMAF activity. At every time point, imuno ${ }^{\circledR}$ showed significantly higher activity in comparison with purified GcMAF and, at $120 \mathrm{~h}$, the activity of imuno ${ }^{\circledR}$ was still well above baseline, whereas GcMAF did not show any residual activity 


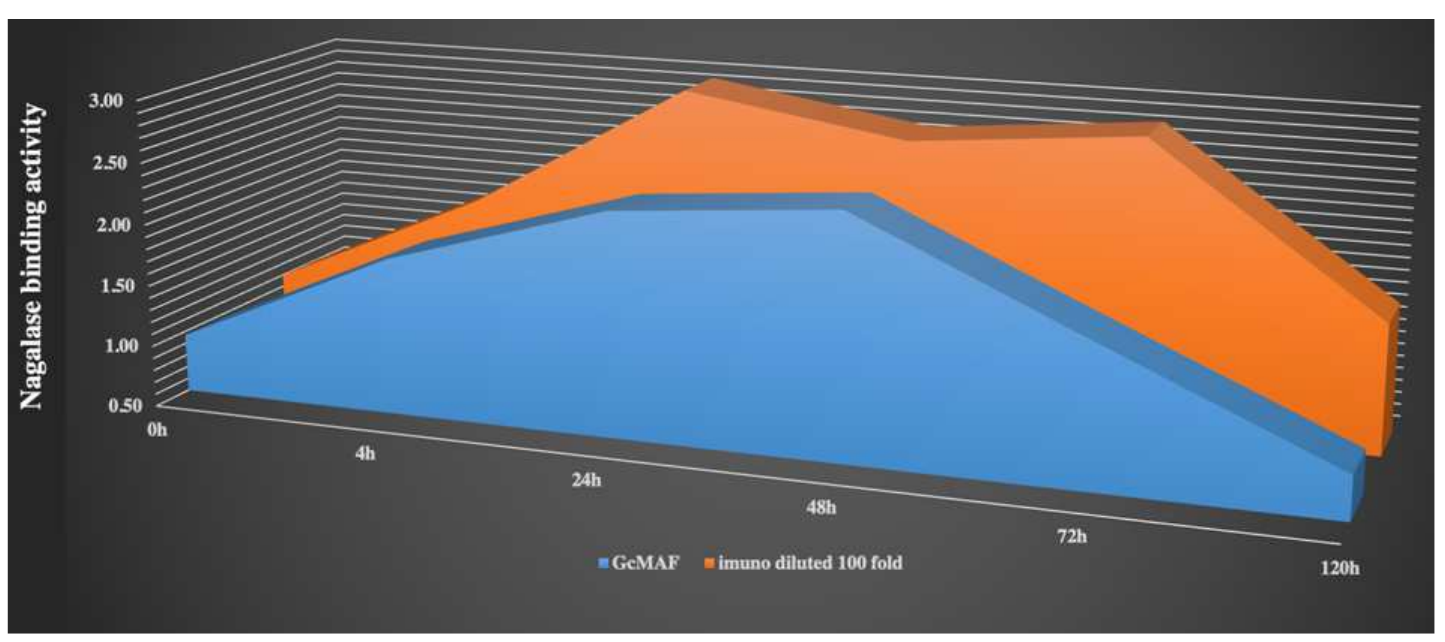

Fig. 4: Kinetics of complex formation between purified GcMAF or imuno ${ }^{\circledR}$ and human nagalase. 3D area representation of data reported in Fig. 3

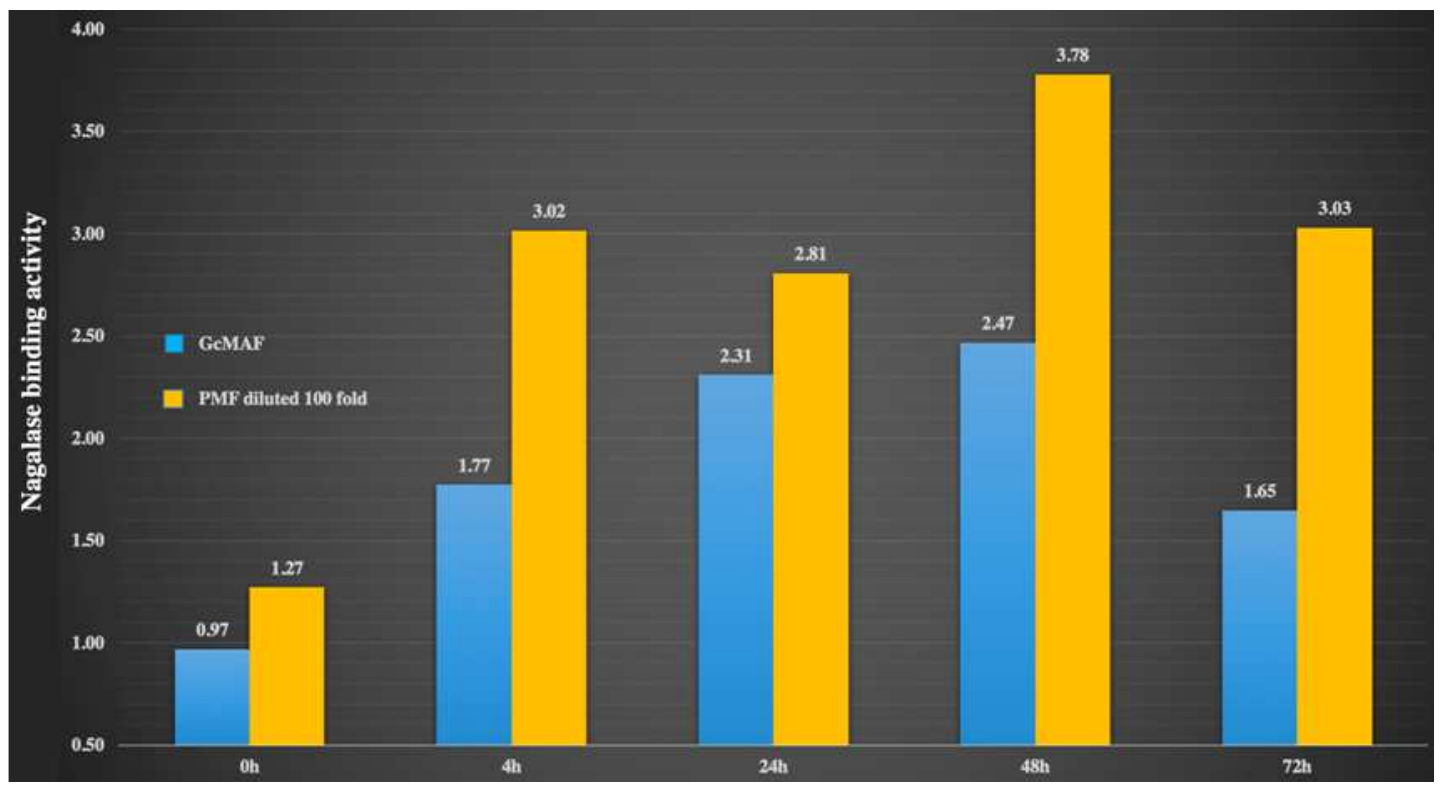

Fig. 5: Kinetics of complex formation between purified GcMAF or Freeze-Dried Bravo Colostrum and Probiotic Complex (PMF) and human nagalase. Values for nagalase complex formation activity in the absence of GcMAF or PMF, with only PBS in the reaction mixture, were taken as 1.00 . PMF, diluted $100 \mathrm{fold}$, had an initial value (at time 0 ) higher than that observed with PBS alone, thus demonstrating immediate, intrinsic GcMAF. At every time point, PMF showed significantly higher activity in comparison with purified GcMAF

Experimental data obtained in vitro and represented in Figs. 3 and 4 demonstrate the GcMAF activity of imuno ${ }^{\circledR}$. Figure 3 shows the kinetics of in vitro interaction between human nagalase and imuno ${ }^{\circledR}$ with resulting formation of nagalase/imuno ${ }^{\circledR}$ complexes detected as described in the Materials and Methods. Since it is well assessed that nagalase specifically binds GcMAF, formation of complexes between nagalase and imuno ${ }^{\circledR}$ is direct demonstration of the GcMAF activity of imuno ${ }^{\circledR}$. As shown in Fig. 3, purified GcMAF, used as positive control, formed complexes with human nagalase only after $4 \mathrm{~h}$ incubation, reached a peak at $48 \mathrm{~h}$ and returned below baseline values at $120 \mathrm{~h}$. These kinetics for purified GcMAF were taken as benchmark. imuno ${ }^{\circledR}$, diluted 100 fold, had an initial value (at time 0) higher than that observed with PBS alone, thus demonstrating immediate, intrinsic GcMAF activity. At every time point, imuno ${ }^{\circledR}$ showed significantly higher activity in comparison with purified GcMAF and, at $120 \mathrm{~h}$, the activity of imuno ${ }^{\circledR}$ was still well above baseline, whereas GcMAF did not show any residual activity. Therefore, it may be argued that the GcMAF activity of imuno $^{\circledR}$ is more than 100 fold higher than that of purified 
GcMAF and more sustained in time. These results are consistent with the in silico observation described above as well as with the fact that chondroitin sulfate, one of the constituents of imuno $^{\circledR}$, has many more alpha-N- acetylgalactosamine moieties per molecule than GcMAF. Figure 4 shows the same results represented as 3D areas in order to highlight the significantly higher GcMAF activity of imuno ${ }^{\circledR}$ as compared to purified GcMAF.

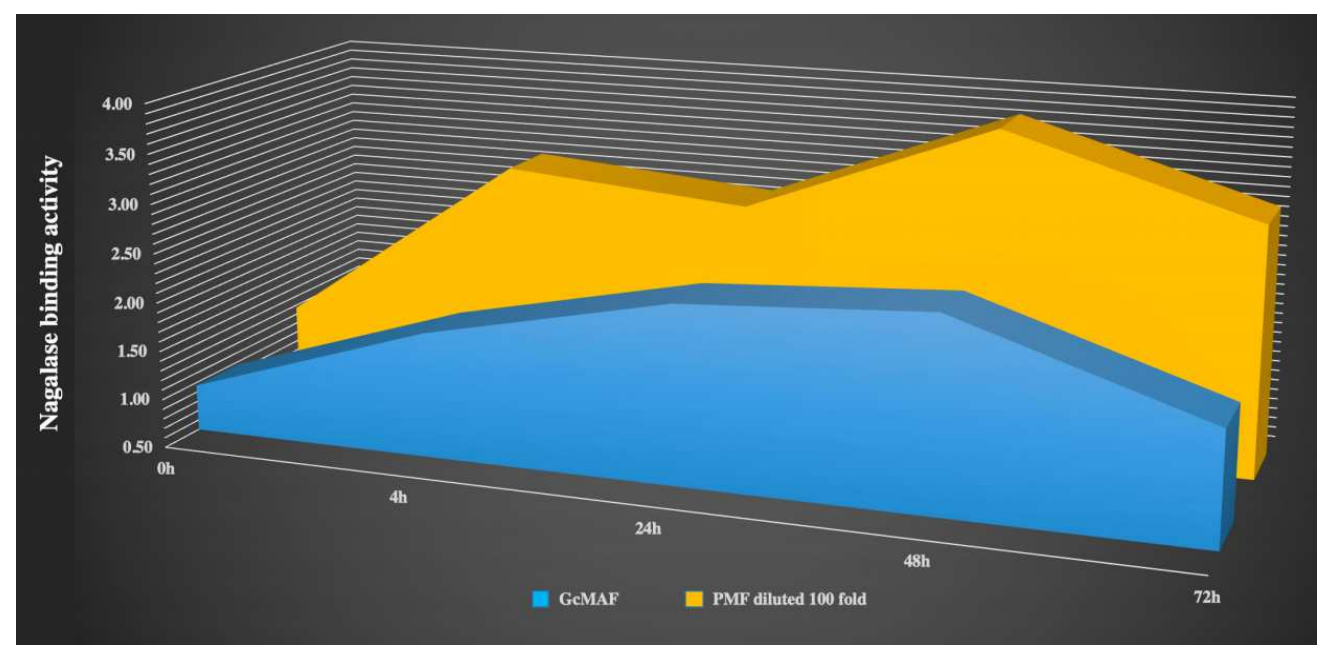

Fig. 6: Kinetics of complex formation between purified GcMAF or Freeze-Dried Bravo Colostrum and Probiotic Complex (PMF) and human nagalase. 3D area representation of data reported in Fig. 5.

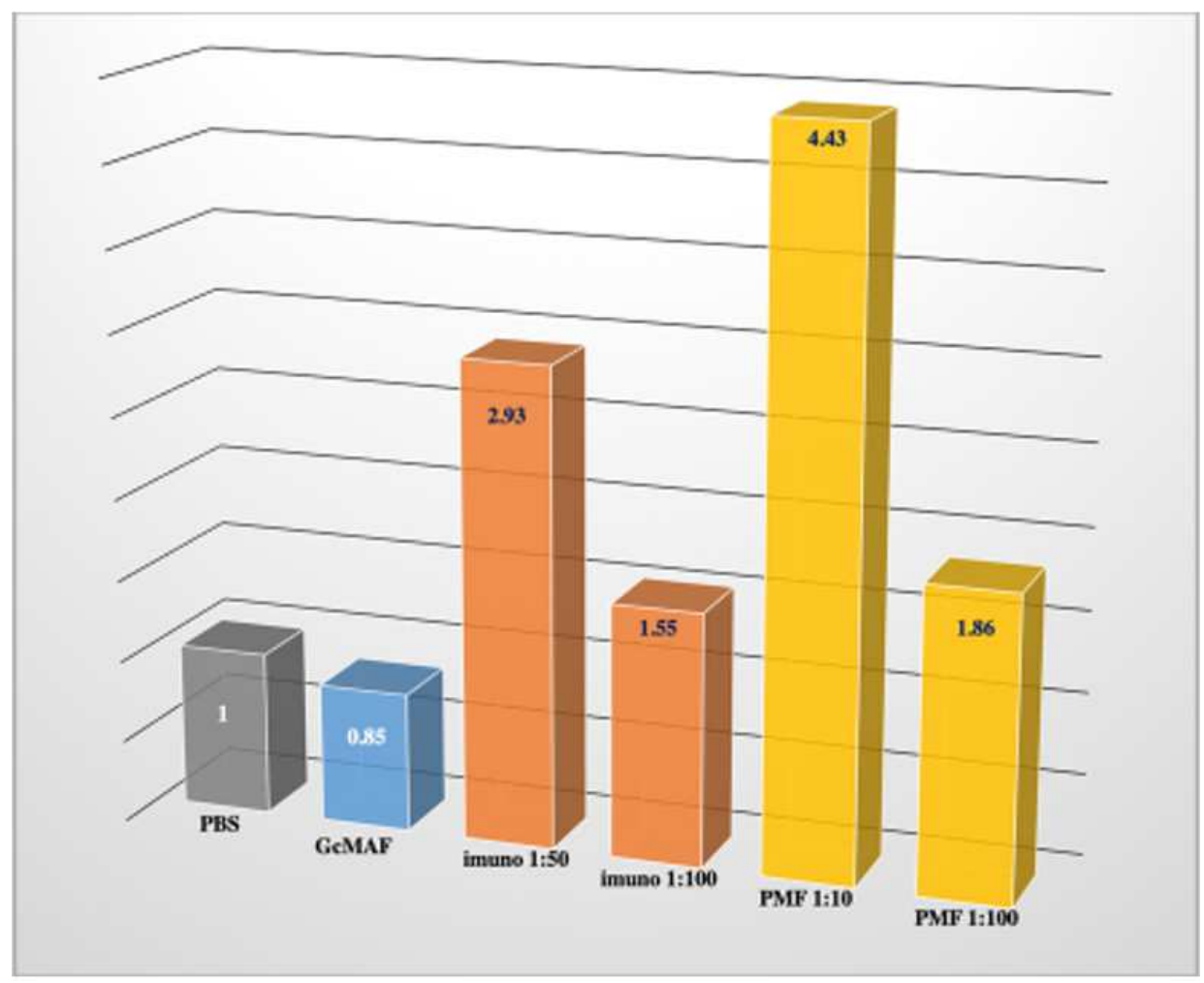

Fig. 7: Kinetics of complex formation between GcMAF, imuno ${ }^{\circledR}$ or Freeze-Dried Bravo Colostrum and Probiotic Complex (PMF) and human nagalase after $120 \mathrm{~h}$ incubation. Values for nagalase complex formation activity in the absence of GcMAF, imuno $^{\circledR}$, or PMF, with only PBS in the reaction mixture, were taken as 1.00. imuno ${ }^{\circledR}$, diluted 1:50 showed higher GcMAF activity than imuno ${ }^{\circledR}$ diluted 1:100 just like PMF, diluted 1:10 was more active than PMF diluted 1:100. imuno ${ }^{\circledR}$ and PMF at the highest dilution (1:100) were significantly more active than control (PBS alone) or purified GcMAF 
Experimental data obtained in vitro and represented in Figs. 5 and 6 demonstrate the GcMAF activity that is present in PMF. Since it is well assessed that nagalase specifically binds GcMAF, formation of complexes between nagalase and PMF is direct demonstration of the presence of active GcMAF in PMF as we described since 2011 (Pacini et al., 2011). Figure 5 and 6 shows the kinetics of in vitro interaction between human nagalase and PMF with resulting formation of nagalase/PMF complexes. In analogy with what observed with imuno ${ }^{\circledR}$, PMF at 1:100 dilution had an initial value (at time 0) higher than that observed with PBS alone, thus demonstrating the presence of immediate, intrinsic GcMAF activity. At every time point, PMF showed significantly higher activity in comparison with purified GcMAF and, at $120 \mathrm{~h}$, the GcMAF activity of PMF was still well above baseline, whereas purified GcMAF did not show any residual activity (Fig. 7). Therefore, it may be argued that the GcMAF activity in PMF is more than 100 fold higher than that of purified GcMAF and more sustained in time. The 100 fold higher activity of PMF in comparison with purified GcMAF can be explained taking into consideration two elements.

1. Naturally formed GcMAF in PMF is associated with vitamin $\mathrm{D}_{3}$, fatty acids and glycosaminoglycans such as chondroitin sulfate - that are normal constituents of milk and colostrum. Since 2013, we proposed a molecular model describing how non-covalent association of these constituents in a multi-molecular complex significantly enhanced the biological activity of GcMAF (Thyer et al., 2013b). This model was independently confirmed by a recent study demonstrating that association with vitamin $D_{3}$ significantly increased the immune stimulating activity of GcMAF (Greilberger and Herwig, 2020).

2. Phages in PMF synthesize proteins with activities superimposable to that of GcMAF that complement the effects of the naturally formed GcMAF that is present in PMF. For example, the protein RAD52 that is the human homolog of the protein Sak encoded by Lactococcus phage ul36, shows significant similarity with the active site of GcMAF. When the sequences of human RAD52 (P43351) and GcMAF (P02774) were obtained and aligned using the Align tool of Uniprot, alignment showed a striking similarity between the active site of GcMAF that is the sequence TPTELAK and the sequence DPAQTSD of RAD52. Since it is well known that phages stimulate the immune system and it is also known that such a stimulation is associated with macrophage activation (Pacini and Ruggiero, 2019a), it is may be hypothesized that GcMAF-like proteins encoded by phages contribute to the overall GcMAF activity of PMF.

Figure 7, shows the formation of nagalase/GcMAF, nagalase/imuno ${ }^{\circledR}$, or nagalase/PMF complexes after $120 \mathrm{~h}$ incubation. It is worth noticing that imuno ${ }^{\circledR}$ diluted 1:50 showed higher GcMAF activity than imuno ${ }^{\circledR}$ diluted 1:100 just like PMF diluted 1:10 was more active than PMF diluted 1:100. These results demonstrate a classic dose-response relationship for both imuno ${ }^{\circledR}$ and PMF as far as their respective GcMAF activity is concerned. In addition, both imuno ${ }^{\circledR}$ and PMF at the highest dilution $(1: 100)$ were significantly more active than the control (PBS alone) or purified GcMAF. It is worth considering that these results were obtained in vitro, that is in the absence of any variable or confounding factor that may affect interpretation of results previously observed in clinical settings (Thyer et al., 2013a; present case report).

\section{Discussion}

The changes observed during the three-month experience here described, support the hypothesis that such a nutritional approach provides health benefits ranging from immune system support to reduction of age-related low-level inflammation. A decrease of serum nagalase activity can be interpreted as the result of modulatory activities of imuno ${ }^{\circledR}$ and PMF on the immune system with particular reference to GcMAF activity and macrophage activation (Ruggiero and Pacini 2018a). As demonstrated here, both imuno ${ }^{\circledR}$ and PMF have intrinsic, direct GcMAF activity according to the results of the experiments in vitro performed by an independent laboratory and described in Figs. 3-7. It is therefore conceivable that the regimen adopted by Dr. MC ( 1 capsule of the probiotic per day and $0.1 \mathrm{~mL}$ of the supplement twice a week) represents the ideal combination to obtain the most benefit from the two complementary approaches. As far as the decrease of CRP is concerned, it is worth noticing that imuno ${ }^{\circledR}$ was designed to optimize mitochondrial function as we described in a recent paper (Pacini and Ruggiero, 2019c) and it is well assessed that aging and chronic conditions are associated with, or possibly caused by, mitochondrial dysfunction that leads to chronic energy imbalance with resulting oxidative stress, systemic inflammation and elevation of CRP values (Gajewski et al., 2017). Therefore, it is plausible that optimization of mitochondrial function by imuno ${ }^{\circledR}$, together with the antiinflammatory properties of PMF thoroughly described in two recent articles (Pacini and Ruggiero, 2019a; 2019b) was responsible for the observed decrease of CRP that was in turn responsible for the ultra-low PINI score recorded at the end of the three-month experience.

The Authors wish to underline the concept that the results here described are from one individual and not part of a wider study. However, the Authors believe the observations like this may benefit those addressing the impact of nutrition on health and successful aging. We consider that this approach is consistent with that 
proposed by Aronson (2005) who encourages reports of single cases in medicine. Since the results observed in this report cannot be generalized at this stage, further studies are warranted to evaluate whether the nutritional approach here described will yield similar results in other subjects either in the context of healthy aging or in the presence of pathologic conditions. These words of caution notwithstanding, it is worth mentioning that the combination of the probiotic and the supplement here described proved very effective in the nutritional approach to a case of multiple myeloma (Antonucci et al., 2019b) thus lending credibility to the hypothesis that such a combination may bring substantial benefits in a number of diverse conditions.

\section{Conclusion}

The experience of Dr. MC suggests that the combination of a novel supplement based on lowmolecular-weight chondroitin sulfate, ultrapure phosphatidylcholine and vitamin $\mathrm{D}_{3}$ (imuno ${ }^{\circledR}$ ) and an extremely diverse probiotic featuring strains with known healthy properties, may lead to overall beneficial effects on health as evidenced by positive changes of indicators of immune system function and healthy aging such as nagalase, CRP and albumin.

\section{Acknowledgement}

The Authors wish to thank Dr. Tanja Mijatovic, PhD, Chief Scientific Officer at R.E.D. Laboratories, for insights on the significance of laboratory tests and for contributing to the understanding of the role of nagalase in health and disease.

\section{Authors' Contributions}

Michael Carter: Performed the experience described in this study, provided critical input and assisted in revising and improving the paper.

Marco Ruggiero and Stefania Pacini: Wrote the first draft of this paper, provided critical input and assisted in revising and improving the paper.

\section{Disclosures}

Michael Carter is Corporate Medical Director of Real Health Clinics, Atlanta, GA, Chief Medical Officer for Health Revival Partners and Chief Medical Advisor for Tissue Regeneration Technologies, Woodstock, GA, USA; he discloses no conflict of interest. He bought all the supplements used during his experience and paid for the analyses reported in this study.

Marco Ruggiero is the founder and CEO of Silver Spring Sagl, Mendrisio, Switzerland, the company producing the probiotic used by Dr. MC. Stefania Pacini works as Quality Control Responsible Person for Silver Spring Sagl. Marco Ruggiero is the inventor of a number of supplements and, together with Stefania Pacini, developed both the probiotic (Freeze- Dried BravoColostrum and Probiotic Complex - PMF) and the supplement (imuno ${ }^{\circledR}$ ) mentioned in this article. Neither Dr. Ruggiero nor Dr. Pacini had prior knowledge of the nutritional plan followed by Dr. MC nor of the results of the analyses that were communicated only after completion of the experience. Likewise, they had no prior knowledge of the results of the experiments with nagalase in vitro that were independently performed by R.E.D. Laboratories (Zellik, Belgium). Marco Ruggiero is member of the Editorial Board of The American Journal of Immunology and is waived from the Article Processing fee for this contribution; he receives no remuneration for his editorial work.

\section{References}

Allin, K.H. and B.G. Nordestgaard, 2011. Elevated Creactive protein in the diagnosis, prognosis and cause of cancer. Crit. Rev. Clin. Lab. Sci., 48: 155-170. DOI: 10.3109/10408363.2011.599831

Antonucci, N., D. Klinghardt, S. Pacini and M. Ruggiero, 2018. Tailoring the Ruggiero-Klinghardt protocol to immunotherapy of autism. Am. J. Immunol., 14: 34-41. DOI: 10.3844/ajisp.2018.34.41

Antonucci, N., S. Pacini and M. Ruggiero, 2019a. Clinical experience of integrative autism treatment with a novel type of immunotherapy. Madridge J. Vaccines, 3: 71-76. DOI: 10.18689/mjv-1000116

Antonucci, N., S. Pacini and M. Ruggiero, 2019b. Use of an extremely biodiverse probiotic and a supplement based on microbial chondroitin sulfate is associated with a significant decrease of serum free kappa light chains as well as a trend toward normalization of kappa/lambda ratio and of plasma cell bone marrow infiltration in a case of multiple myeloma. Am. J. Immunol., 15: 5-9. DOI: 10.3844/ajisp.2019.5.9

Arima, H., M. Kubo, K. Yonemoto, Y. Doi and T. Ninomiya et al., 2008. High-sensitivity C-reactive protein and coronary heart disease in a general population of Japanese: The Hisayama study. Arterioscler. Thromb. Vasc. Biol., 28: 1385-1391. DOI: 10.1161/ATVBAHA.107.157164

Aronson, J.K., 2005. Unity from diversity: The evidential use of anecdotal reports of adverse drug reactions and interactions. J. Eval. Clin. Pract., 11: 195-208. DOI: 10.1111/j.1365-2753.2005.00527.x

Bradstreet, J.J., E. Vogelaar and L. Thyer, 2012. Initial observations of elevated alpha-Nacetylgalactosaminidase activity associated with autism and observed reductions from Gc proteinMacrophage Activating Factor injections. Autism Insights, 4: 31-38. DOI: 10.4137/AUI.S10485 
Breiterman-White, R., 2006. C-reactive protein and anemia: Implications for patients on dialysis. Nephrol. Nurs. J., 33: 555-558. PMID: 17044439

Brown, A.S., A. Sourander, S. Hinkka-Yli-Salomäki, I.W. McKeague and J. Sundvall et al., 2014. Elevated maternal C-reactive protein and autism in a national birth cohort. Mol. Psychiatry, 19: 259-264. DOI: $10.1038 / \mathrm{mp} .2012 .197$

Caines, M.E., H. Zhu, M. Vuckovic, L.M. Willis and S.G. Withers et al., 2008. The structural basis for T-antigen hydrolysis by Streptococcus pneumoniae: A target for structure-based vaccine design. J. Biol. Chem., 283: 31279-3183. DOI: 10.1074/jbc.C800150200

Cozlea, D.L., D.M. Farcas, A. Nagy, L.M. Willis and S.G. Withers et al., 2013. The impact of $\mathrm{C}$ reactive protein on global cardiovascular risk on patients with coronary artery disease. Curr. Health Sci. J., 39: 225-231.PMID: 24778862

Dowling, P.C. and S.D. Cook, 1976. Disease markers in acute multiple sclerosis. Arch Neurol., 33: 668-670. DOI: 10.1001/archneur.1976.00500100002003

Fabris, A., P. Biagioni, T. Punzi, G. Morucci and M. Gulisano et al., 2012. Role of angiotensinconverting enzyme and vitamin $\mathrm{d}$ receptor gene polymorphisms in cancer anorexia-cachexia syndrome. Am. J. Immunol., 8: 65-70.

DOI: 10.3844 /ajisp.2012.65.70

Ferrucci, L., A. Corsi, F. Lauretani, S. Bandinelli and B. Bartali et al., 2005. The origins of age-related proinflammatory state. Blood, 105: 2294-2299. DOI: 10.1182/blood-2004-07-2599

Gajewski, M, P. Rzodkiewicz and S. Maśliński, 2017. The human body as an energetic hybrid? New perspectives for chronic disease treatment? Reumatologia, 55: 94-99. DOI: 10.5114/reum.2017.67605

Ghorpade, D.S., L. Ozcan, Z. Zheng, S.M. Nicoloro and Y. Shen et al., 2018. Hepatocyte-secreted DPP4 in obesity promotes adipose inflammation and insulin resistance. Nature, 555: 673-677.

DOI: 10.1038 /nature26138

Greco, M., M.D. Mitri, F. Chiriacò, G. Leo and E. Brienza et al., 2009. Serum proteomic profile of cutaneous malignant melanoma and relation to cancer progression: Association to tumor derived alpha-Nacetylgalactosaminidase activity. Cancer Lett., 283: 222-229. DOI: 10.1016/j.canlet.2009.04.001

Greilberger, J. and R. Herwig, 2020. Vitamin Ddeglycosylated vitamin $\mathrm{d}$ binding protein dimer: Positive synergistic effects on recognition, activation, phagocytosis and oxidative stress on macrophages. Clin. Lab. DOI: 10.7754/Clin.Lab.2019.191121

Havre, P.A., M. Abe, Y. Urasaki, K. Ohnuma and C. Morimoto et al., 2008. The role of CD26/dipeptidyl peptidase IV in cancer. Front Biosci., 13: 1634-1645. DOI: $10.2741 / 2787$
Hiromura, M., K. Nohtomi, Y. Mori, H. Kataoka and M. Sugano et al., 2018. Caveolin-1, a binding protein of CD26, is essential for the anti-inflammatory effects of dipeptidyl peptidase- 4 inhibitors on human and mouse macrophages. Biochem. Biophys. Res. Commun., 495: 223-229. DOI: 10.1016/j.bbrc.2017.11.016

Korbelik, M., V.R. Naraparaju and N. Yamamoto, 1998. The value of serum alpha-N-acetylgalactosaminidase measurement for the assessment of tumour response to radio- and photodynamic therapy. Br. J. Cancer, 77: 1009-1014. DOI: 10.1038/bjc.1998.166

McFarland, D.C., K. Shaffer, W. Breitbart, B. Rosenfeld and A.H. Miller, 2019. C-reactive protein and its association with depression in patients receiving treatment for metastatic lung cancer. Cancer, 125: 779-787. DOI: $10.1002 /$ cncr.31859

Oluleye, O.W., A.R. Folsom, V. Nambi, P.L. Lutsey and C.M. Ballantyne, 2013. 2013. Troponin T, B-type natriuretic peptide, C-reactive protein and causespecific mortality. Ann. Epidemiol., 23: 66-73.

DOI: 10.1016/j.annepidem.2012.11.004

Pacini, S., T. Punzi, G. Morucci and M. Ruggiero, 2011. Macrophages of the Mucosa- Associated Lymphoid Tissue (MALT) as key elements of the immune response to vitamin $\mathrm{d}$ binding protein- macrophage activating factor. It. J. Anat. Embryol., 116: 136-136. DOI: $10.13128 / \mathrm{IJAE}-10160$

Pacini, S. and M. Ruggiero, 2019a. Phage composition of a fermented milk and colostrum product assessed by microbiome array; putative role of open reading frames. bioRxiv.

Pacini, S. and M. Ruggiero, 2019b. Natural plasmids in a Swiss fermented milk and Colostrum product assessed by Microbiome Array. Madridge J. Immunol., 3: 100-108. DOI: 10.18689/mjim-1000123

Pacini, S. and M. Ruggiero, 2019c. Color Doppler evaluation of isovolumetric relaxation time and of signals arising from axons of the median nerve as a means to evaluate mitochondrial functionality in the context of immunotherapy of cancer and chronic conditions associated with mitochondrial dysfunction. Am. J. Immunol., 15: 22-32. DOI: 10.3844 /ajisp.2019.22.32

Pro, B. and N.H. Dang, 2004. CD26/dipeptidyl peptidase IV and its role in cancer. Histol. Histopathol., 19: 1345-1351. DOI: $10.14670 / \mathrm{HH}-19.1345$

Puzianowska-Kuźnicka, M., M. Owczarz, K. Wieczorowska-Tobis, P. Nadrowski and J. Chudek et al., 2016. Interleukin-6 and C-reactive protein, successful aging and mortality: The PolSenior study. Immun Age., 13: 21-21. DOI: $10.1186 / \mathrm{s} 12979-016-0076-\mathrm{x}$

Ruggiero, M. and S. Pacini, 2018a. Rationale for the design of a novel tool for immunotherapy based on an emulsion of glycosaminoglycan. Integr. Cancer Sci. Therap., 5: 1-5 DOI: 10.15761/ICST.1000285 
Ruggiero, M. and S. Pacini, 2018b. A novel potential adjuvant for cancer vaccines. Madridge J. Vaccines, 2: 57-61. DOI: $10.18689 / \mathrm{mjv}-1000112$

Saburi, E., J. Tavakol-Afshari, S. Biglari and Y. Mortazavi, 2017. Is $\alpha-\mathrm{N}$-acetylgalactosaminidase the key to curing cancer? A mini-review and hypothesis. J. BUON, 22: 1372-1377. PMID: 29332325

Schwalb, M., M. Taubmann, S. Hines, H. Reinwald and M. Ruggiero, 2016. Clinical observation of a novel, complementary, immunotherapeutic approach based on ketogenic diet, chondroitin sulfate, vitamin $\mathrm{d} 3$, oleic acid and a fermented milk and colostrum product. Am. J. Immunol., 12: 91-98. DOI: 10.3844 /ajisp.2016.91.98

Tang, Y., E. Fung, A. Xu and H.Y. Lan, 2017. Creactive protein and ageing. Clin. Exp. Pharmacol. Physiol., 44: 9-14. DOI: 10.1111/1440-1681.12758

Thyer, L., E. Ward, R. Smith, J.J.V. Branca and G. Morucci et al., 2013a. GC protein-derived macrophage-activating factor decreases $\alpha-\mathrm{N}$ acetylgalactosaminidase levels in advanced cancer patients. Oncoimmunology, 2: e25769-e25769. DOI: 10.4161/onci.25769

Thyer, L., E. Ward, R. Smith, R. Smith and M.G. Fiore et al., 2013b. A novel role for a major component of the vitamin D axis: Vitamin D binding proteinderived macrophage activating factor induces human breast cancer cell apoptosis through stimulation of macrophages. Nutrients, 5: 2577-2589.

DOI: $10.3390 /$ nu5072577
Weaving, G., G.F. Batstone and R.G. Jones, 2016. Age and sex variation in serum albumin concentration: An observational study. Ann. Clin. Biochem., 53: 106-111. DOI: $10.1177 / 0004563215593561$

Yamamoto, N. and M. Urade, 2005. Pathogenic significance of alpha-N-acetylgalactosaminidase activity found in the hemagglutinin of influenza virus. Microbes Infect., 7: 674-681. DOI: $10.1016 /$ j.micinf.2005.01.015

Yamamoto, N., 2006. Pathogenic significance of alpha-Nacetylgalactosaminidase activity found in the envelope glycoprotein gp160 of human immunodeficiency virus Type 1. AIDS Res. Hum Retroviruses, 22: 262-271. DOI: 10.1089 /aid.2006.22.262

Yamamoto, N. and V.R. Naraparaju, 1997. Immunotherapy of BALB/c mice bearing Ehrlich ascites tumor with vitamin D-binding proteinderived macrophage activating factor. Cancer Res., 57: 2187-2192. PMID: 9187119

Zunaid, I.R., S. Pacini and M. Ruggiero, 2020. Significance of hydrophobic and charged sequence similarities in sodium-bile acid cotransporter and vitamin D-binding protein macrophage activating factor. bioRxiv. 